\title{
USING PERIODICITY TO MITIGATE GROUND VIBRATION
}

\author{
Lars Vabbersgaard Andersen \\ Aalborg University, Department of Civil Engineering \\ Sofiendalsvej 9-11, DK-9200 Aalborg SV, Denmark \\ e-mail: la@civil.aau.dk
}

Keywords: Wave mitigation, wave impeding block, WIB, finite element analysis, FEA.

\begin{abstract}
Introduction of trenches, barriers and wave impeding blocks on the transmission path between a source and receiver can be used for mitigation of ground vibration. However, to be effective a barrier must have a depth of about one wavelength of the waves to be mitigated. Hence, while great reductions of energy transmission can be obtained within the medium and high frequency ranges, barriers are a poor solution at low frequencies.

As an alternative, a periodic change in the properties of the soil can be introduced-to the geometry and/or to the material. Periodic structures are known to act as filters for wave propagation, effectively reducing the transmission of energy in certain frequency bands known as stop bands or band gaps, thus only allowing propagation in the so-called pass bands.

In this paper, a stratified ground with two soil layers is considered and two types of periodicity is analysed: A soil with periodic stiffening (ground improvement) and a ground with periodic changes in the surface elevation obtained by artificial landscaping. By means of a two-dimensional finite-element model, the stiffness and mass matrices are determined for a single cell of the ground with horizonal periodicity. Floquet analysis is then performed in order to quantify the number of propagating wave modes as well as modes with low degrees of attenuation. As a conclusion of the analysis, effective mitigation in the low frequency range can be established. The position of stop bands can be manipulated by engineering the soil with periodic changes to the geometry and material.
\end{abstract}




\section{INTRODUCTION}

Ground vibration from traffic or unbalanced machinery is a nuisance to people living and working next to railways, roads or production facilitiesespecially in cases with poor sub grades where waves can propagate over long distances. Further, sensitive equipment in laboratories and hospitals may malfunction due to even small vibration levels. Different methods for mitigation of ground vibration have been studied and tested, ranging from ground improvement and use of trenches or barriers [1] to application of damping or isolation devices.

This paper proposes a different approach, namely the use of periodic shaping of the landscape, inspired by the curvy hills and valleys formed around the Max IV laboratory near Lund, Sweden. Originally intended by the architects as an aesthetic improvement of the environment, Persson [2] found in his licentiate work that the modification of the terrain is able to provide ground vibration mitigation.

The idea of using periodic structures for mitigation of vibrations is not new. In the classical work by Mead [3], wave propagation in a periodic one-dimensional waveguide was studied, and many others have followed the same idea. Simple problems can be analysed analytically by means of Floquet analysis, and combining this approach with, for example, the finite-element method, periodic two and three-dimensional structures of complex geometries can be studied regarding their wave propagation properties. Such models have been used, for example, by Manconi and Mace [4] to study curved panels, and by Domadiya et al. [5] to study wave propagation in beams with periodically placed masses or changes to the cross section. The combination of Floquet theory and finite-elements has also be used for analysis of railways [6], mainly to allow studying long structures with low computational cost. For a similar reason, Bian et al. [7] used a so-called 2.5D model to study ground vibrations from a train running on a track with irregular surface.

In the present work, the wave propagation in a soil layer is analysed by combining the finiteelement method with Floquet theory. A reference cell, i.e. one period of the periodic ground geometry, is modeled using the commercial finite-element analysis software Abaqus 6.14 [8]. The stiffness and mass matrices are then extracted, and an in-house code is utilized for Floquet analysis. The wave characteristics of the soil including periodicity are discussed in terms of propagating, attenuating and evanescent modes, and pass and stop bands for vibration transmission are identified. Two different types of ground are examined. Firstly, a layer with periodically changing material properties is considered, corresponding to a case with jet grouting or another type of ground improvement implemented with equidistant spacing. Secondly, inspired by the Max IV laboratory, a ground with periodic changes in the surface geometry is concerned.

\section{COMPUTATIONAL MODEL}

\subsection{Finite-element formulation for soil with periodic geometry}

Wave propagation in the layered soil is analysed by a two-dimensional finite-element model assuming plane strain and linear elastic response. The geometry is periodic in the direction along the ground surface-i.e. a reference "cell" is repeated infinitely many times in the $x$ direction as illustrated in Figure 1. In the absence of damping, the equation of motion for the finite-element model of the cell can be written in the time domain as

$$
\mathbf{M} \ddot{\mathbf{u}}(t)+\mathbf{K u}(t)=\mathbf{f}(t),
$$

where $\mathbf{M}$ and $\mathbf{K}$ are the mass and stiffness matrices, respectively, whereas $\ddot{\mathbf{u}}(t)$ and $\mathbf{u}(t)$ are the nodal acceleration and displacement vectors, respectively, and $\mathbf{f}(t)$ is the vector of external 
nodal forces. After Fourier transformation with respect to time, $t$, Eq. (1) may be written in frequency domain:

$$
\left(\mathbf{K}+(\mathrm{i} \omega)^{2} \mathbf{M}\right) \mathbf{U}(\omega)=\mathbf{D}(\omega) \mathbf{U}(\omega)=\mathbf{F}(\omega), \quad \mathbf{D}(\omega)=\mathbf{K}-\omega^{2} \mathbf{M}
$$

where $\mathrm{i}=\sqrt{-1}$ is the imaginary unit and $\omega$ signifies the angular frequency, whereas $\mathbf{U}(\omega)$ and $\mathbf{F}(\omega)$ are the complex-valued Fourier coefficients of the displacement and external force vectors, respectively. Finally, $\mathbf{D}(\omega)$ is introduced as the complex-valued dynamic stiffness matrix.

In what follows, all calculations are expressed in the frequency domain and for convenience the dependence of the angular frequency $\omega$ is assumed implicit. For example, $\mathbf{U}(\omega)=\mathbf{U}$. The next step is to split the dynamic stiffness matrix as well as the displacement and force vectors into components associated with internal degrees of freedom and components associated with degrees of freedom at the boundary where periodicity constraints are to be applied. Introducing subscripts $i$ and $b$ to identify degrees of freedom for internal nodes and periodicity boundary nodes, respectively, sub-matrices and sub-vectors are defined as:

$$
\mathbf{D}=\left[\begin{array}{ll}
\mathbf{D}_{b b} & \mathbf{D}_{b i} \\
\mathbf{D}_{i b} & \mathbf{D}_{i i}
\end{array}\right], \quad \mathbf{U}=\left\{\begin{array}{c}
\mathbf{U}_{b} \\
\mathbf{U}_{i}
\end{array}\right\}, \quad \mathbf{F}=\left\{\begin{array}{l}
\mathbf{F}_{b} \\
\mathbf{F}_{i}
\end{array}\right\} .
$$

It is noted that the internal degrees of freedom include those at nodes on parts of the boundary that are not subject to periodicity conditions. Inserting Eq. (3) into Eq. (2) and assuming that no external forces are applied to the interior degrees of freedom provides:

$$
\left[\begin{array}{ll}
\mathbf{D}_{b b} & \mathbf{D}_{b i} \\
\mathbf{D}_{i b} & \mathbf{D}_{i i}
\end{array}\right]\left\{\begin{array}{l}
\mathbf{U}_{b} \\
\mathbf{U}_{i}
\end{array}\right\}=\left\{\begin{array}{l}
\mathbf{F}_{b} \\
\mathbf{F}_{i}
\end{array}\right\}=\left\{\begin{array}{c}
\mathbf{F}_{b} \\
\mathbf{0}_{i}
\end{array}\right\}
$$

Here $\mathbf{0}_{i}$ is the null vector with the same dimensions as $\mathbf{F}_{i}$.

By condensation of the internal degrees of freedom it follows that

$$
\tilde{\mathbf{D}} \mathbf{U}_{b}=\mathbf{F}_{b}, \quad \tilde{\mathbf{D}}_{b b}=\mathbf{D}_{b b}-\mathbf{D}_{b i} \mathbf{D}_{i i}^{-1} \mathbf{D}_{i b},
$$

where $\tilde{\mathbf{D}}$ is a dynamic stiffness matrix for the cell, expressed in terms of boundary degrees of freedom only. For the present problem, $\tilde{\mathbf{D}}$ is much smaller than the original dynamic stiffness matrix for the cell, D, given a small number of boundary nodes compared to internal nodes.

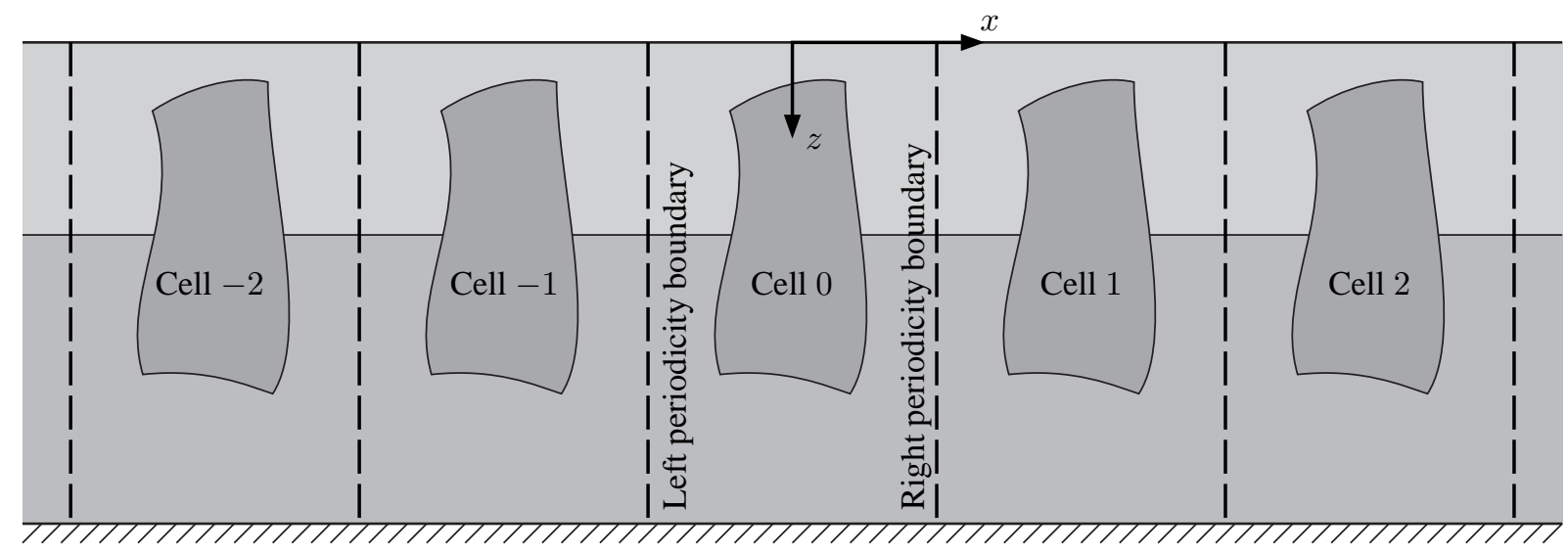

Figure 1: Periodic ground with two layers over bedrock and repeated inclusion of an object. 
With reference to Figure 1, the boundary nodes are divided into those belonging to the left periodicity boundary and those belonging to the right periodicity boundary, using the subscripts $L$ and $R$, respectively. Equation (5) can then be expressed into the form

$$
\left[\begin{array}{cc}
\tilde{\mathbf{D}}_{L L} & \tilde{\mathbf{D}}_{L R} \\
\tilde{\mathbf{D}}_{R L} & \tilde{\mathbf{D}}_{R R}
\end{array}\right]\left\{\begin{array}{c}
\mathbf{U}_{L} \\
\mathbf{U}_{R}
\end{array}\right\}=\left\{\begin{array}{c}
\mathbf{F}_{L} \\
\mathbf{F}_{R}
\end{array}\right\}
$$

where the sub-matrices $\tilde{\mathbf{D}}_{L L}, \tilde{\mathbf{D}}_{L R}, \tilde{\mathbf{D}}_{R L}$ and $\tilde{\mathbf{D}}_{R R}$ are all square matrices since the number of nodes and degrees of freedom must be the same at the left and right boundaries of the cell. Likewise, $\mathbf{U}_{L}, \mathbf{U}_{R}, \mathbf{F}_{L}$ and $\mathbf{F}_{R}$ are column vectors of the same length. Otherwise, periodicity constraints cannot be applied.

The next step is to apply periodicity conditions. Based on Floquet theory, the following continuity and equilibrium condiotions must hold for the displacements and forces, respectively, at the left and right periodicity boundaries:

$$
\mathbf{U}_{R}=\exp (-\mathrm{i} k \Delta) \mathbf{U}_{L}=\lambda \mathbf{U}_{L}, \quad \exp (-\mathrm{i} k \Delta) \mathbf{F}_{L}+\mathbf{F}_{R}=\lambda \mathbf{F}_{L}+\mathbf{F}_{R}=\mathbf{0}_{L}
$$

Here $\mathbf{0}_{L}$ is the null vector having the same length as $\mathbf{F}_{L}$ and $\mathbf{F}_{R}$. Further, $k$ is the complexvalued wavenumber related to a given mode of vibration or propagation, $\Delta$ is the length of the cell, i.e. the distance between the left and right boundaries, and $\lambda=-\mathrm{i} k \Delta$. Propagating modes without attenuation are characterised by real-valued wavenumbers and $|\lambda|=1$.

Equation (7) can be reformulated into the form

$$
\begin{gathered}
\mathbf{U}_{b}=\left\{\begin{array}{l}
\mathbf{U}_{L} \\
\mathbf{U}_{R}
\end{array}\right\}=\left[\begin{array}{c}
\mathbf{I} \\
\lambda \mathbf{I}
\end{array}\right] \mathbf{U}_{L}=\boldsymbol{\Lambda}_{R} \mathbf{U}_{L}, \quad \boldsymbol{\Lambda}_{R}=\left[\begin{array}{c}
\mathbf{I} \\
\lambda \mathbf{I}
\end{array}\right], \\
{\left[\begin{array}{ll}
\lambda \mathbf{I} & \mathbf{I}
\end{array}\right]\left\{\begin{array}{l}
\mathbf{F}_{L} \\
\mathbf{F}_{R}
\end{array}\right\}=\boldsymbol{\Lambda}_{L} \mathbf{F}_{b}=\mathbf{0}_{L}, \quad \boldsymbol{\Lambda}_{L}=\left[\begin{array}{ll}
\lambda \mathbf{I} & \mathbf{I}
\end{array}\right],}
\end{gathered}
$$

where $\mathbf{I}$ is the identity matrix with the same dimensions as $\tilde{\mathbf{D}}_{L L}$. Pre-multiplying Eq. (5) by $\boldsymbol{\Lambda}_{L}$ and making use of the results obtained in Eq. (8) lead to an equation in the form

$$
\boldsymbol{\Lambda}_{L} \tilde{\mathbf{D}} \boldsymbol{\Lambda}_{R} \mathbf{U}_{L}=\boldsymbol{\Lambda}_{L} \mathbf{F}_{b}=\mathbf{0} \Rightarrow[\lambda \mathbf{I} \quad \mathbf{I}]\left[\begin{array}{cc}
\tilde{\mathbf{D}}_{L L} & \tilde{\mathbf{D}}_{L R} \\
\tilde{\mathbf{D}}_{R L} & \tilde{\mathbf{D}}_{R R}
\end{array}\right]\left[\begin{array}{c}
\mathbf{I} \\
\lambda \mathbf{I}
\end{array}\right] \mathbf{U}_{L}=\mathbf{0}_{L}
$$

which can be expressed as a non-linear eigenvalue problem in terms of the eigenvalues $\lambda$ and the eigenvectors $\mathbf{U}_{L}$ :

$$
\left(\lambda^{2} \tilde{\mathbf{D}}_{L R}+\lambda\left(\tilde{\mathbf{D}}_{L L}+\tilde{\mathbf{D}}_{R R}\right)+\tilde{\mathbf{D}}_{R L}\right) \mathbf{U}_{L}=\mathbf{0}_{L}
$$

This may conveniently be reformulated into a generalized linear eigenvalue problem of the doubled dimensions,

$$
\left(\left[\begin{array}{cc}
\mathbf{0}_{L L} & \tilde{\mathbf{D}}_{L L}+\tilde{\mathbf{D}}_{R R} \\
\tilde{\mathbf{D}}_{L R} & \tilde{\mathbf{D}}_{L L}+\tilde{\mathbf{D}}_{R R}
\end{array}\right]-\lambda\left[\begin{array}{cc}
\tilde{\mathbf{D}}_{L L}+\tilde{\mathbf{D}}_{R R} & \mathbf{0}_{L L} \\
\mathbf{0}_{L L} & -\tilde{\mathbf{D}}_{L R}
\end{array}\right]\right)\left\{\begin{array}{c}
\mathbf{U}_{L} \\
\mathbf{U}_{R}
\end{array}\right\}=\left\{\begin{array}{l}
\mathbf{0}_{L} \\
\mathbf{0}_{L}
\end{array}\right\}
$$

where $\mathbf{0}_{L L}$ is the null matrix with the same dimensions as $\tilde{\mathbf{D}}_{L L}$. It is observed that the eigenvector corresponds to the boundary displacement vector $\mathbf{U}_{b}$. Once the eigenvalue problem (11) has been solved, i.e. $\lambda$ and $\mathbf{U}_{b}$ have been determined for a given angular frequency $\omega$, the internal degrees of freedom are determined as $\mathbf{U}_{i}=-\mathbf{D}_{i i}^{-1} \mathbf{D}_{i b} \mathbf{U}_{b}$, which follows directly from Eq. (4). 


\subsection{Geometry and material properties for the models}

Two different periodic soil geometries are considered: a top layer with periodically changing material properties (Model I), and a top layer with periodically changing position of the ground surface (Model II). The overall geometry of the reference cells for the two cases are illustrated in Figure 2. The first case may be considered as periodic ground improvement or wave impeding blocks (WIBs) obtained by, for example, jet grouting. The second case can be obtained by artificial landscaping. The curved ground surface is a combination of circular arcs. The concave and convex parts each constitute one half of the cell length with the convex arc being placed at the centre and the two concave parts constituting one quarter each in the left and right sides, respectively. As shown in Figure 2, a second layer is present below the topsoil in either case, separated from the upper layer by a horizontal interface. Further, it is noted that both cells are symmetrical around the $z$ axis.

Models I and II are characterized by the following parameters: width of the cell, $w_{1}$; width of the WIB, $w_{2}$ (only Model I); depth of the top layer, $h_{1}$; depth of the bottom layer, $h_{2}$; amplitude of the surface elevation, $h_{3}$ (only Model II). Each material is defined by Young's modulus, $E$, Poisson's ratio, $\nu$, and mass density, $\rho$. In the present analysis, the properties listed in Table 1 have been utilized. The properties correspond to a top layer of medium stiff clay, a bottom layer of till, and a WIB made of weak concrete. At the bottom layer is fixed in the $x$ and $z$ directions at its base. This corresponds to a stratified soil over bedrock.

For the finite-element analysis of the master cell, the commercial code Abaqus/Standard [8] has been employed, using Python scripting to allow parametric studies. Quadrilateral elements with eight nodes, quadratic interpolation of the displacement and full integration are applied in all models. A structured mesh is utilized with a global mesh size of $1.0 \mathrm{~m}$, employing the intrinsic mesh generator in Abaqus.

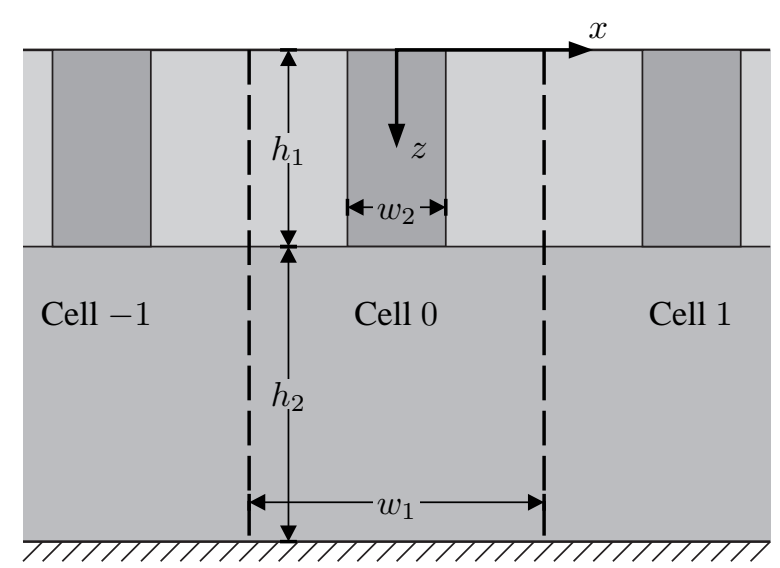

(a) Changing material properties

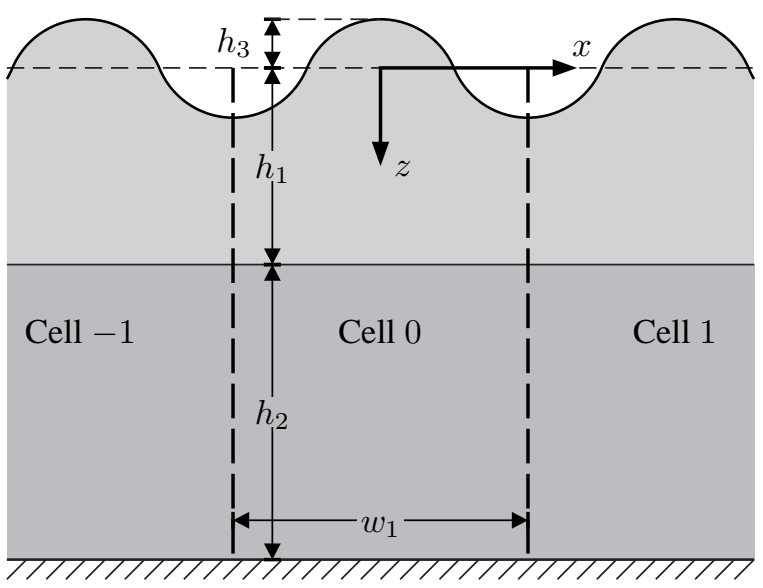

(b) Changing ground surface elevation

Figure 2: Geometry of the two cell configurations considered in the analysis.

Table 1: Material properties.

\begin{tabular}{lccc}
\hline Material & $\begin{array}{c}\text { Young's modulus } \\
E[\mathrm{MPa}]\end{array}$ & $\begin{array}{c}\text { Poisson's ratio } \\
\nu[-]\end{array}$ & $\begin{array}{c}\text { Mass density } \\
\rho\left[\mathrm{kg} / \mathrm{m}^{3}\right]\end{array}$ \\
\hline Top layer soil & 50 & 0.48 & 2000 \\
Bottom layer soil & 500 & 0.45 & 2200 \\
Stiff WIB material & 5000 & 0.20 & 2400 \\
Soft WIB material & 5 & 0.20 & 1200 \\
\hline
\end{tabular}




\section{ANALYSES AND DISCUSSION}

In the following, results are presented and discussed for the two cases presented as Models I and II in the previous section.

\subsection{Model I: Periodic inclusions (wave impeding blocks)}

Figure 3 shows the results for Model I with different cell lengths and wave impeding blocks made of stiff material as defined in Table 1. The figure indicates the number of propagating modes (in one direction only) that exist at frequencies in the range $0-20 \mathrm{~Hz}$. Further, the numbers of attenuating and evanescent modes (decaying in one direction only) have been plotted. Modes with absolute values of the imaginary parts of the wavenumber higher than $1.0 \mathrm{~m}^{-1}$, corresponding to high degrees of attenuation, have been filtered out. Hence, only propagating
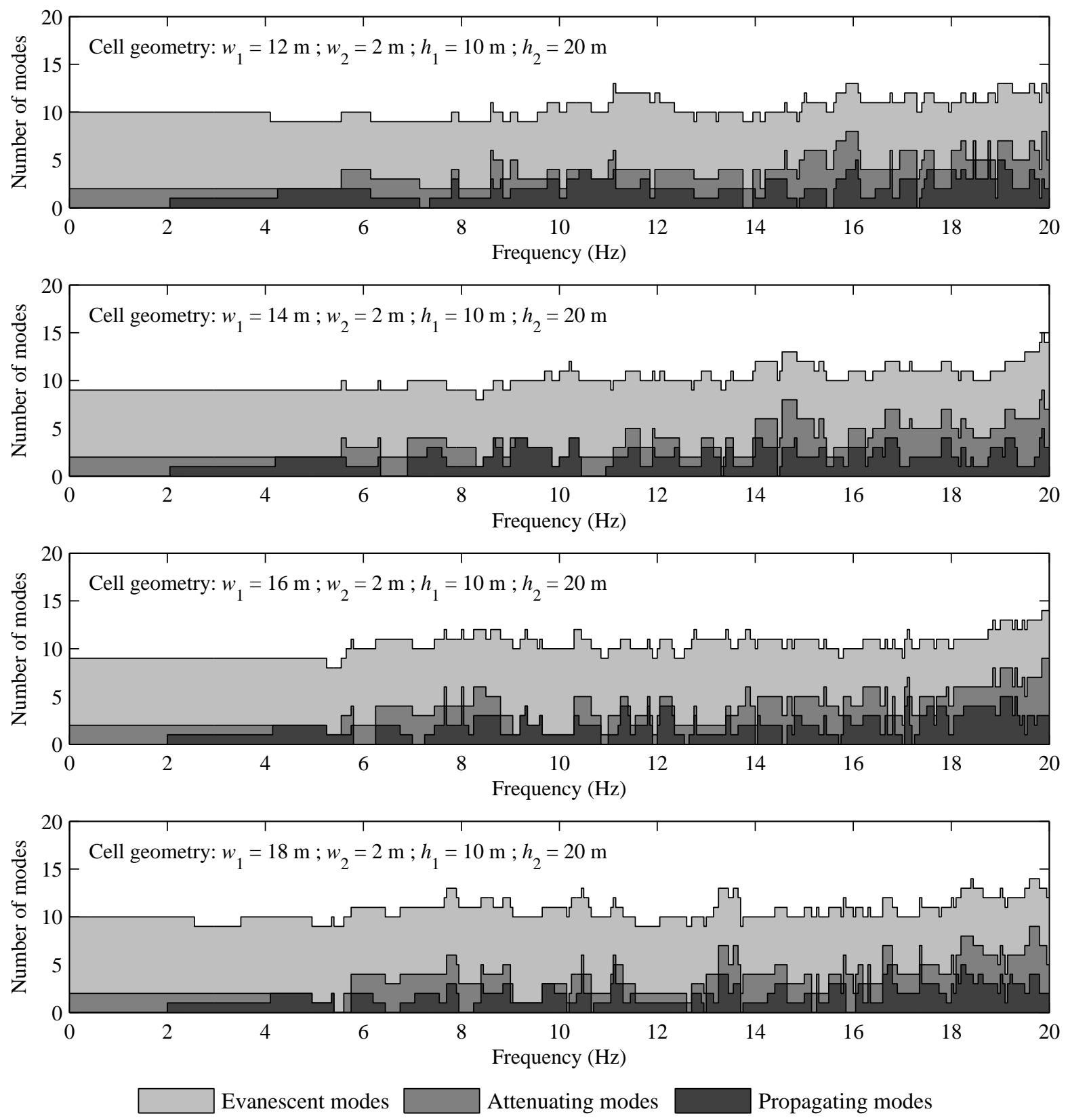

Figure 3: Skyline plots for Model I with different geometries: Stiff WIB material. 
modes and evanescent or attenuating modes with low damping are included in the plots which have been given the name "skyline plots" due to their visual appearance.

For all values of $w_{1}$ included in the study, a number of stop bands are present in the frequency range $0-20 \mathrm{~Hz}$, though most of the stop bands are narrow. Among the tested configurations, the cell width of $14 \mathrm{~m}$ provides the better solution in terms of wave mitigation. Here, relatively wide band gaps are present around $6.5 \mathrm{~Hz}$ and $10.5 \mathrm{~Hz}$. Figuratively, these band gaps can be considered "parks" where the "noise of the city" diminishes.

Results of Model I with soft WIB material are plotted in Figure 4. Evidently, the soft WIBs provide an overall better solution in terms of mitigation. Especially it is noticed that a marked band gap occurs at frequencies of around 7 to $8 \mathrm{~Hz}$, depending on the width of the cell and only for $w_{1}=14$ to $18 \mathrm{~m}$. This stop band has the interesting feature that not only propagating modes but also attenuating modes disappear, leading to absolute tranquility at these frequencies.
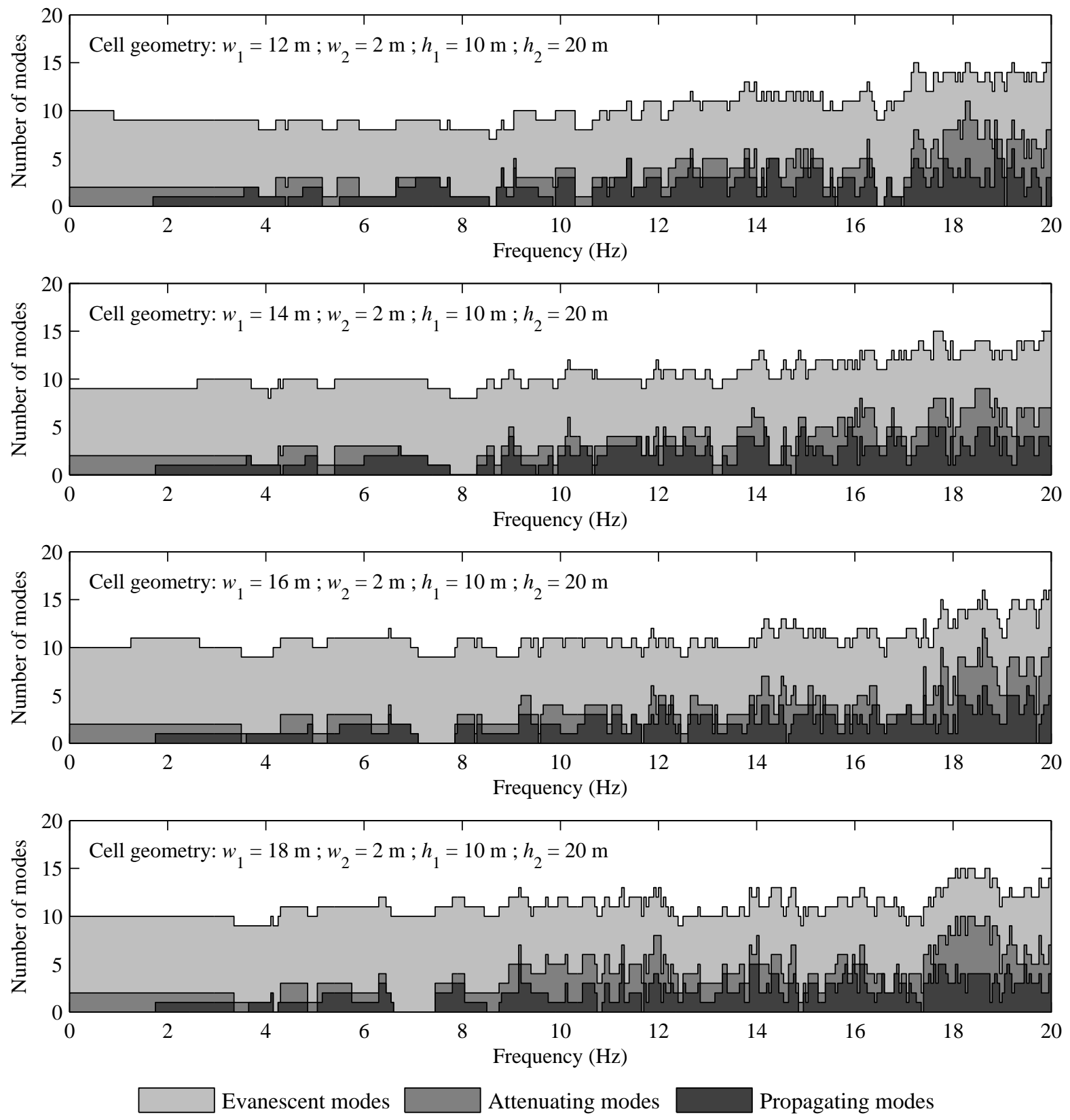

Figure 4: Skyline plots for Model I with different geometries: Soft WIB material. 


\subsection{Model II: Curved landscape}

Figures 5 and 6 contain results of Model II with two different surface elevations, i.e. $h_{3}=1 \mathrm{~m}$ and $h_{3}=2 \mathrm{~m}$. Again, modes with absolute values of the imaginary parts of the wavenumber higher than $1.0 \mathrm{~m}^{-1}$ are disregarded. Cell lengths smaller than about $8 \mathrm{~m}$ have been found to be ineffective for mitigation purposes. However, the cell length should neither be too large as this will make it difficult to obtain a sufficient number of repetitions to provide the required near-periodicity. Due to these reasons, cell lengths of 8,10,12 and $14 \mathrm{~m}$ have been analysed, and as in Model I it is observed that a number of stop bands occur.

Based on the results presented in Figure 5, the cells with $w_{1}=10,12$ or $14 \mathrm{~m}$ perform almost equally well for the surface elevation $h_{3}=1 \mathrm{~m}$, but the band gaps occur at different frequencies. This should be taken into consideration when the ground surface is engineering
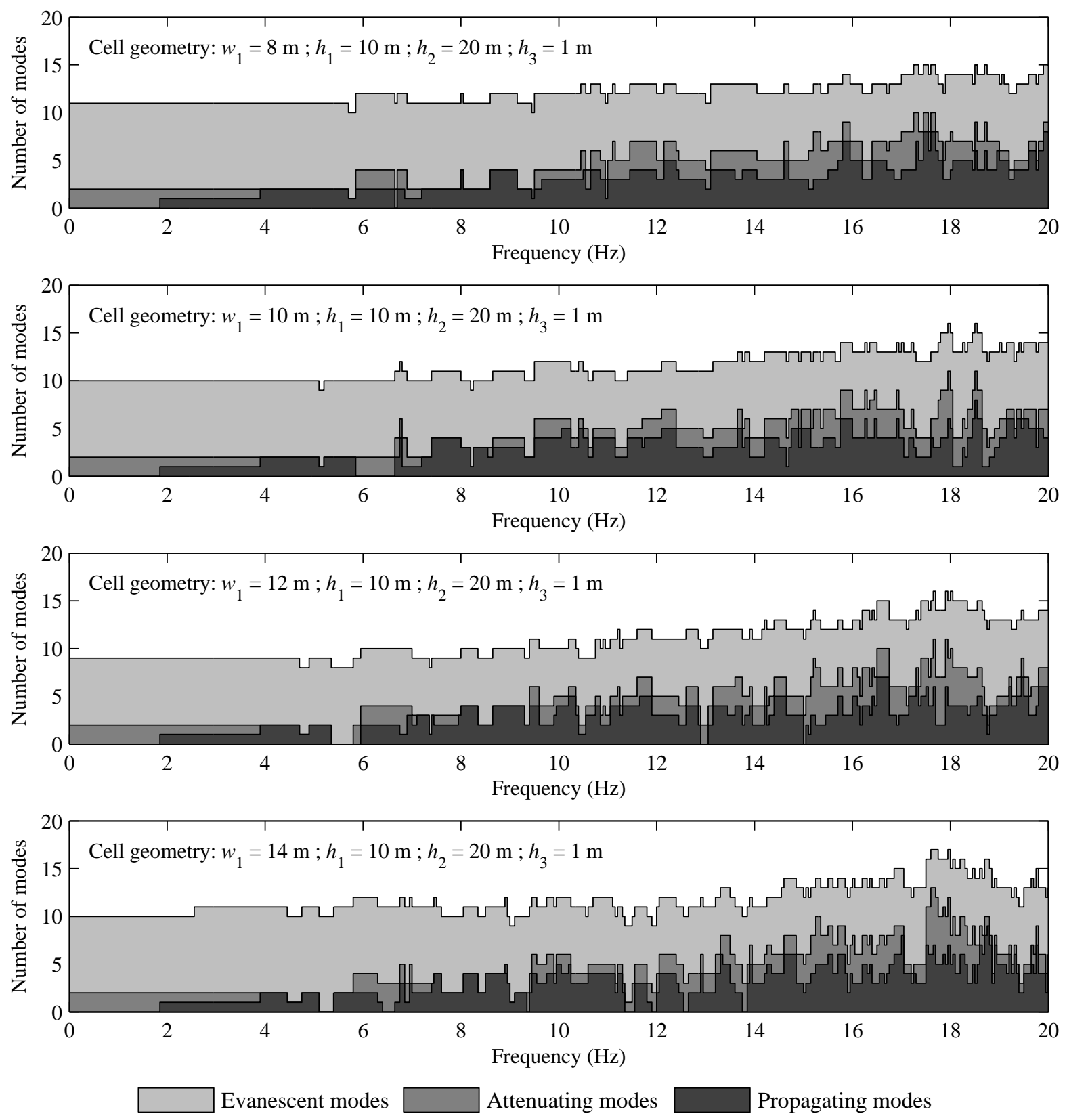

Figure 5: Skyline plots for Model II with different geometries: Surface elevation $h_{3}=1 \mathrm{~m}$. 
to prevent wave propagation a certain frequencies. On the other hand, when $h_{3}=2 \mathrm{~m}$ (cf. Figure 6) the cells with $w_{1}=12$ and $14 \mathrm{~m}$ have a superior performance. Here, a relatively wide range of frequencies is covered by two closely space band gaps around 5 and $6.5 \mathrm{~Hz}$.

\section{Discussion}

The skyline plots presented in Figures 3-6 provide an intuitive approach to understanding how many propagating, attenuating and evanescent modes exist at a given frequency. The target of vibration mitigation is to reduce the height of the "buildings" in the plot, especially the front row which quantifies the number of propagating modes. It must be mentioned that the number of propagating modes should not be influenced by the discretization of the finite-element model of the cell, given that the solution has converged. Similarly, the number of lightly damped
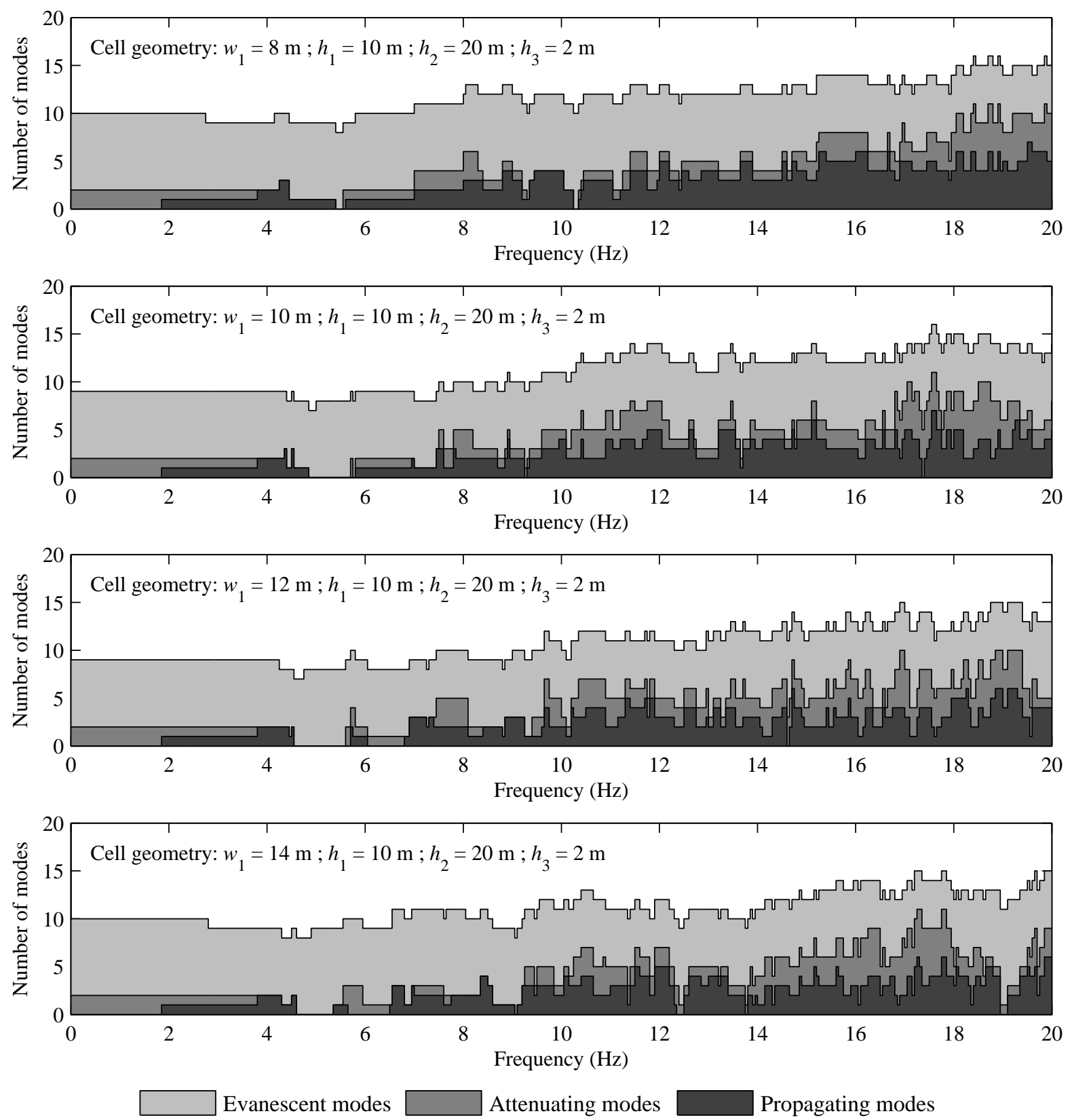

Figure 6: Skyline plots for Model II with different geometries: Surface elevation $h_{3}=2 \mathrm{~m}$. 
attenuating and evanescent modes will not be influenced, i.e. only the number of highly damped modes will increase as a result of mesh refinement.

Comparing the results of Models I and II, it is observed that stop bands of similar width can be achieved by either concept of periodicity for the layered soil considered in the present examples. A cell length of 14-18 m has the higher efficiency for Model I, whereas a cell length of about $10-12 \mathrm{~m}$ is optimal for Model II. It must be noticed that this conclusion is only valid for the stratification considered in the present case, i.e. with the material properties listed in Table 1 and with the layer depths $h_{1}=10 \mathrm{~m}$ and $h_{2}=20 \mathrm{~m}$.

From an installation point of view, the main advantage of Model I is that the ground surface does not need to be changed, i.e. it can remain plane. The main disadvantage is that new material (stiff or soft WIB material) needs to be put into the top layer by grouting or another technique. For Model II it is the opposite. No new material is needed-it is simply a relocation of material from one place to another-but the ground surface elevation is changed by the artificial landscaping. Thus, Model II is interesting for sites where no buildings, roads or other structures are going to be placed directly on the ground as, for example, the site surrounding the Max IV facility near Lund in Sweden [2].

Regarding the frequency range that can effectively be influenced by the periodic inclusions of wave impeding blocks or artificial landscaping, the present analyses indicate that mitigation can be achieved at frequencies well below $10 \mathrm{~Hz}$. Here the wavelength of shear waves in the topsoil is more than $9 \mathrm{~m}$ and typically, single barriers with depths less than one Rayleigh wavelength are ineffective. Finally, it should be emphasized that the first modes of resonance in buildings typically occur at frequencies around $4-6 \mathrm{~Hz}$, and modes of individual floors may occur at frequencies below 10-15 Hz. Consequently, the method of wave mitigation presented in this paper can be attractive-especially for facilities with sensitive equipment or people (e.g. hospitals and laboratories) in the vicinity of railways or heavily trafficked roads.

\section{CONCLUSIONS}

Mitigation of wave propagation in layered soil can effectively be achieved by periodic inclusion of stiff or soft wave impeding blocks (WIBs) or by altering the ground surface in a periodic manner. Based on the combined finite-element and Floquet analysis presented in this paper, the following conclusions can be made:

- Stop bands for wave propagation can be obtained at frequencies as low as $5 \mathrm{~Hz}$;

- To obtain an efficient solution, the cell length must be about 10-12 $\mathrm{m}$ for a solution with periodic changes in the ground surface elevation, whereas for the WIB-based solution a slightly longer cell (14-18 $\mathrm{m}$ in length) has been found optimal in the considered case;

- A "skyline plot" has been suggested as an intuitive way of illustrating the modal density related to propagating, attenuating and evanescent modes in strata.

It should be noted that real soil layers are not perfectly homogeneous and horizontal. Hence, the performance of a periodic change to the top layer of natural soils is subject to great uncertainty. But if the entire top layer is engineered, which may be the case at sites like the Max IV facility, mitigation of low-frequency ground vibration can be achieved.

Finally, the conclusions drawn in the present paper are only valid for the considered strata which consists of a $10 \mathrm{~m}$ deep top layer of soft clayey soil on a $20 \mathrm{~m}$ deep bottom layer of till over bedrock. Future investigations should include other stratifications, and expansion to a three-dimensional model should is relevant to study the efficiency regarding point sources. 


\section{REFERENCES}

[1] L. Andersen and S.R.K. Nielsen, Reduction of ground vibration by means of barriers or soil improvement along a railway track. Soil Dynamics and Earthquake Engineering, 25 (7-10), 701-716.

[2] P. Persson, Reduction in ground vibrations by the use of wave obstacles. Licentiate Dissertation, Department of Construction Sciences, Lund University, Sweden, 2013.

[3] D.J. Mead, Free wave propagation in periodically supported, infinite beams. Journal of Sound and Vibration, 11, 181-197, 1970.

[4] E. Manconi and B.R. Mace, Wave characterization of cylindrical and curved panels using a finite element method. Journal of Acoustical Society of America, 125 (1), 154-163, 2009.

[5] P.G. Domadiya, E. Manconi, M. Vanali, L.V. Andersen, and Andrea Ricci, Numerical and experimental investigation of stop-bands in finite and infinite periodic one-dimensional structures. Journal of Vibration and Control, Published online before print June 25, 2014, doi: $10.1177 / 1077546314537863$.

[6] G. Degrande, D. Clouteau, R. Othman, M. Arnst, H. Chebli, R. Klein, P. Chatterjee, and B. Janssens, A numerical model for ground-borne vibrations from underground railway traffic based on a periodic finite elementboundary element formulation. Journal of Sound and Vibration, 293 (3-5), 645-666, 2006.

[7] X. Bian, C. Chao, W. Jin, and Y. Chen, A 2.5D finite element approach for predicting ground vibrations generated by vertical track irregularities. Journal of Zhejiang University SCIENCE A, 12(12), 885-894, 2011.

[8] Abaqus 6.14. Dassault Systèmes Simulia Corp., Providence, RI, USA. 